\title{
Akute Infektionen nach Osteosynthese
}

\author{
Dieter Nast-Kolb, Sascha Flohé, Steffen Ruchholtz
}

\section{Zusammenfassung}

In Abhängigkeit von der Lokalisation und der durchgeführten Osteosynthese muss in bis zu $9 \%$ mit Infektionen gerechnet werden, wobei in über 80\% Staphylokokken nachgewiesen werden. Dies bedeutet ein strenges postoperatives Überwachungsmanagement, um diese Komplikationen so früh wie möglich zu diagnostizieren und therapieren. Schmerzverlauf, klinischer Lokalbefund, CRP- und Blutbildbestimmung sowie die Sonographie stellen die postoperative Standarddiagnostik dar. Jeder sich erhärtende Verdacht stellt eine dringende Indikation zur operativen Revision dar. Um die Erreger- und Resistenzbestimmung nicht zu behindern sollte die Antibiotikatherapie mit Clindamycin + Rifampicin oder Oflaxacin im- mer erst intraoperativ nach Abstrichund Gewebeabnahme erfolgen. Therapeutisch steht in der Regel ein mehrzeitiges operatives Debridement mit ausgiebiger Spülung im Vordergrund. Lässt sich dabei keine ausreichende Weichteildeckung erreichen, so sind plastisch-rekonstruktive Maßnahmen indiziert. In Abhängigkeit von der Lokalisation, der Dauer und dem Ausmaß der Infektion sowie der Stabilität der Osteosynthese kann eine Implantatbelassung möglich oder ein Verfahrenswechsel erforderlich sein. Im Intervall kommt der Vakuumversiegelung eine herausragende Bedeutung zu. Mit einem standardisierten Infektionsmanagement lässt sich heute in den meisten Fällen eine erfolgreiche Behandlung der Komplikation ohne zusätzliche Beeinträchtigung der Funktionalität erreichen.

\section{Einleitung}

Durch die zunehmend individuellere operative Versorgungsmöglichkeit bez. Frakturform u. -lokalisation ist eine immer bessere Rekonstruktion und Rehabilitation auch nach komplexen Verletzungen möglich. Mit dem Ziel der Wiederherstellung von Gelenkfunktionen und schnellen Belastbarkeit ist die operative Therapie verglichen mit konservativen Behandlungsmethoden entscheidend überlegen. Der einzige relevante Nachteil ist jedoch das operationsbedingte Infektionsrisiko. Eine postoperative Infektion stellt, wenn nicht im absoluten Frühstadium erkannt, auch heute noch für den betroffenen Patienten eine erheblich verlängerte Behandlungsdauer mit ungewis-

OP-JOURNAL 2004; 20: 86-91

(c) Georg Thieme Verlag KG Stuttgart · New York sem Heilungsausgang und für die Gesellschaft ein Vielfaches der Kosten dar.

Kampf et al. [12] fanden für das Jahr 1994 in ihrem gesamtchirurgischen Krankenkollektiv von 3373 Patienten eine postoperative Infektionsrate von 2,63\%, diese

Tab. 1 Infektionshäufigkeit in Abhängigkeit von der Lokalisation [2, 3, 6, 9, 10, 13, 16, 17, 19]

\begin{tabular}{lll} 
Lokalisation & Osteosynthese & $\%$ Infekte \\
\hline Oberarm & Platte/Nagel & $0 \%$ \\
Unterarm & Platte & $0,7 \%$ \\
Wirbelsäule & gemischt & $4,1 \%$ \\
Proximaler Femur & Nagel/DHS & $0,9-3,6 \%$ \\
Femurschaft (offen + geschlossen) & Platte/Nagel/Fixateur & $0-9 \%$ \\
Proximale Tibia & Fixateur/Platte & $5,5 \%$ \\
Tibiaschaft (offen + geschlossen) & Platte/Nagel/Fixateur & $0-7,8 \%$ \\
OSG/Pilon (offen +geschlossen) & Platte/Fixateur ext. & $-26 \%$ \\
Calacaneus & Platte & $1,7-3,1 \%$
\end{tabular}

betrug dabei für die Gesamtheit aller 562 Osteosynthesen $2,80 \%$.

Ein genaueres Bild vermittelt die Inzidenz für eine Frühinfektion nach Osteosynthese in Abhängigkeit von Körperregion, Frakturtyp und Osteosyntheseverfahren. Bei einer diesbezüglich durchgeführten Medline-Analyse mit den Schlagwörtern „osteosynthesis“ und „infection“ der Jahre 1984-2003 ergibt sich aus den relevanten Untersuchungen eine Streubreite von 0 bis über $25^{\circ}$ (Tab.1).

\section{Diagnose und Therapie des Frühinfektes}

Vor diesem Hintergrund kommt der Früherkennung eines gestörten Heilungsverlaufes eine außerordentliche Bedeutung zu.

Dabei ist zwischen einer hämatombedingten drohenden oder manifesten Frühinfektion zu unterscheiden.

\section{Definition}

Die akute Infektion nach einer Osteosynthese, welche auch als akute postoperative Osteitis bezeichnet wird, beschreibt eine Infektion des Implantatlagers und der heilenden Fraktur innerhalb von 8 Wochen nach Trauma und operativer Ver- 
sorgung. Später manifest werdende Infektionen werden als chronische Infekte bezeichnet, wobei manche Autoren die Grenze nach 3 Monaten ziehen [11,18].

\section{Infekt-Diagnostik}

Bei jedem postoperativen Verlauf ist während der ersten Tage eine tägliche lokale Befundkontrolle und -dokumentation obligat.

Erster Hinweis auf eine drohende oder sich anbahnende Infektion ist eine über den 2./3. postoperativen Tag hinaus persistierende lokale Beschwerdesymptomatik. Die Wundsituation zeigt eine perifokale Rötung, sie kann aber auch inspektorisch weit gehend unauffällig sein. Daneben muss eine anhaltende Wundsekretion sowie gespannte lokale Weichteile an eine sich anbahnende Infektion denken lassen. Bei persistierender Sezernierung zeigt sich zunächst die regelmäßige Durchfeuchtung der Wundverbände mit klarer oder bernsteinfarbener Flüssigkeit. Im weiteren Verlauf kommt es im Falle einer Infektion zur putriden Verfärbung des Wundsekrets.

Bei angrenzenden Gelenken müssen auch diese mit denselben Kriterien in die Diagnostik einbezogen werden. Dabei ist insbesondere auf eine postoperativ oder sich sekundär entwickelnde Ergussbildung zu achten, welche steril abpunktiert und bei jeglichem Infektverdacht bakteriologisch untersucht werden muss.

In der laborchemischen Untersuchung hat die wiederholt durchgeführte CRPBestimmung den höchsten Stellenwert: Postoperativ persistierend hohe bzw. sekundär wieder ansteigende CRP-Werte müssen immer als Hinweis auf ein lokales oder differenzial diagnostisch systemisch-entzündliches Geschehen gewertet werden. Eine Leukozytose tritt dagegen häufig erst nachgeschaltet bei manifester Infektsituation auf.

Zeichen der drohenden Infektion bzw. Frühinfektion:

- anhaltende oder neu aufgetretene Schmerzen

— lokale Rötung oder Überwärmung

- anhaltende Sekretion

- gespannte lokale Weichteile

- anhaltende oder neu auftretende CRPErhöhung bzw. Leukozytose

- Temperaturerhöhung

Diagnostisch stellt neben der klinischen und der engmaschigen laborchemischen
Kontrolle die Ultraschalluntersuchung mit Frage eines Flüssigkeitsverhaltes eine entscheidende Maßnahme dar.

Jeder Nachweis eines größeren Hämatoms stellt im Zusammenhang mit den oben genannten klinischen und laborchemischen Auffälligkeiten eine drohende Infektion mit der Konsequenz der unverzüglichen operativen Revision dar. Bei positivem Keimnachweis handelt es sich per definitionem um eine Frühinfektion.

\section{Erregernachweis}

In den meisten Fällen (ca. 75\%) werden implantatassoziierte Infekte durch Staphylokokken-Arten verursacht. Aerobe gramnegative Bakterien finden sich in $10-20 \%$ und anaerobe Bakterien in weiteren $10 \%$ der Fälle $[5,15]$. In über $60 \%$ der Infekte mit positivem Keimnachweis lassen sich durch extensive Untersuchungsund Bebrütungsverfahren (siehe unten) Mischinfektionen mit mehreren Keimen nachweisen [15].

Es kann jedoch mitunter schwierig sein, im Rahmen eines üblichen Abstrichs oder Punktates bei normaler Bebrütung über 48-72 Stunden einen sicheren Keimnachweis zu erbringen. So wird bei chronischen Gelenkinfekten nach Endoprothesen durch die üblichen Verfahren nur in 40-60\% der Fälle ein Erreger nachgewiesen [15]. Diese niedrige Nachweisrate wird im Allgemeinen bedingt durch [1]:

- die geringe Keimzahl im Gebiet der Protheseninfektion

- den niedrigen Metabolismus mancher Erreger

- eine bereits eingeleitete Antbiotikatherapie (z.B. durch vorbehandelnde Ärzte)

Um die Nachweisrate bei postoperativen Infektionen zu erhöhen, sollten auch für die Infektionen nach Osteosynthesen folgende Punkte berücksichtigt werden, welche für infizierte Endoprothesen aufgezeigt wurden [15]:

- längerfristige Bebrütung des Probenmaterials (über 7 Tage)

a intraoperative Entnahme und bakteriologische Untersuchung von infiziertem Gewebe (anstelle von alleinigen Abstrichen)

- zusätzliche Untersuchung von Abkratzpräparaten der Implantatoberfläche (Biofilm!) - dies gilt jedoch insbesondere für ein bereits länger bestehendes Infektionsbestehen.
Neut [15] konnte dabei nachweisen, dass sich durch diese intensivierte Keimsuche die Rate an positiven Erregernachweisen auf $86 \%$ erhöhen lässt.

\section{Therapiekonzepte}

Wie oben ausgeführt, sollte bei drohenden Infektionen bzw. Frühinfektionen umgehend eine operative Revision erfolgen.

Das Ausmaß des operativen Vorgehens richtet sich nach Art und Ausdehnung des Infektes, der Lokalisation, der lokalen Weichteilsituation sowie der Stabilität der durchgeführten Osteosynthese und beinhaltet ein radikales Debridement mit ausgiebiger Spülung, die Erhaltung bzw. Wiederherstellung der Stabilität sowie die Gewährleistung einer vitalen Weichteildeckung.

Das operative Vorgehen wird von einer intraoperativ begonnenen adjuvanten Antibiotikatherapie begleitet.

\section{Debridement, Spülung}

Der Zugang erfolgt über die Wunde der initialen Osteosynthese. Dabei wird die Narbe mit dem darunter gelegenen subkutanen Gewebe vollständig exzidiert.

In den tieferen Schichten müssen kompromisslos sämtliche avitalen Faszienund Weichteilgewebsanteile entfernt werden.

Gleichzeitig gilt es, dabei unter Kenntnis der Vaskularität gewebeschonend zu präparieren und wenn möglich, Kompartimentgrenzen zu beachten.

Dabei ist unbedingt eine zusätzliche Denudierung des Knochens durch Ablösung von Periost und/oder Knochen zu vermeiden.

Liegt jedoch avitaler Knochen ohne gut durchblutete Weichteildeckung vor, so muss dieser ebenfalls sorgfältig debridiert werden, ohne Rücksicht auf dadurch eventuell entstehende Knochendefekte.

Eine Sonderform stellt die infizierte Marknagelung dar, wo das Knochendebridement durch ein großzügiges Aufbohren des Markraumes erreicht werden kann.

Nach dem Debridement stellt die ausgiebige Spülung den zweiten entscheidenden Behandlungsschritt dar. 
Die Spülung kann in Form einer Jet-Lavage durchgeführt werden. Der Vorteil liegt in der Möglichkeit, die Wundhöhle mit großen Mengen an Flüssigkeit (z.B. 5 Liter) optimal in kurzer Zeit in allen Winkeln zu reinigen.

Eine Saugspüldrainage kann nur in Ausnahmen indiziert sein, wie z. B. bei nach Debridement einer Markraumphlegmone.

\section{Spülflüssigkeiten}

Bei der Verwendung von lokalen Spülflüssigkeiten ist zu berücksichtigen, dass Povidon-Jod-haltige Lösung (Betaisadona) ihre antiseptische Wirkung in Anwesenheit von Proteinen verlieren. Von einigen Autoren wird die Spülung mit dem Antiseptikum Lavasept $t^{\circledR}$ angege- ben, ohne dass dazu kontrollierte Studien vorliegen. Von den meisten Autoren wird deshalb die lokale Spülung mit steriler Kochsalzlösung empfohlen.

\section{Stabilität der Osteosynthese}

Die Stabilität stellt eine unabdingbare Voraussetzung jeder Knochenbruchund Infektionsheilung dar.

Bei der Frühinfektion nach Osteosynthese liegt immer eine instabile ossäre Situation vor. In Abhängigkeit von der Lokalisation, vom Ausmaß und der anzunehmenden Zeitdauer der Infektion sowie der Durchblutung und Weichteilldeckung ist zu entscheiden, ob eine Implantatbelassung möglich oder ein Verfahrenswechsel erforderlich ist.
So kann sicher stabilisierendes Osteosynthesematerial dann belassen werden, wenn die ossäre Durchblutung der betroffenen Region und eine vitale Weichgewebedeckung gewährleistet sind.

Dies gilt insbesondere für anatomisch rekonstruierte Gelenkfrakturen, bei Ausschluss einer infektiösen Gelenkbeteiligung, aber auch für stabilisierende Verfahren nach instabilen Verletzungen der Wirbelsäule. In dieser Situation würde die Auflösung der primären Osteosynthese neben der sicher nicht gleichwertigen alternativen Stabilisierung auch ein erhöhtes Risiko der intraartikulären bzw. intraspinalen Infektionsverschleppung beinhalten (Abb.1).

Bei diesem „Implantat erhaltenden Vorgehen“ ist jedoch immer zu berücksichti-
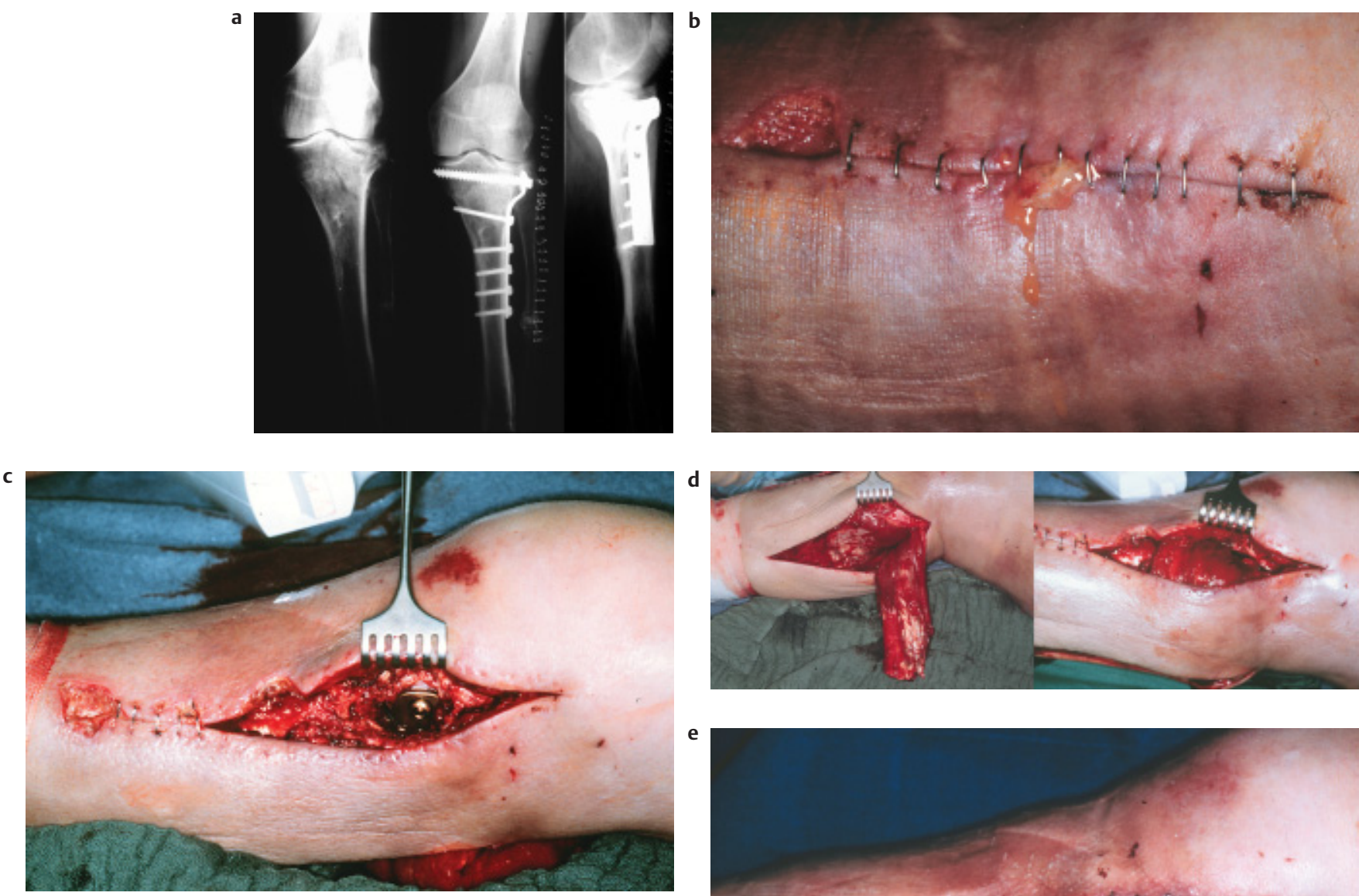

Abb.1 Implantatbelassung bei Frühinfektion 9 Tage nach TibiakopfAbstützplatte. (a) Röntgen prä- und postoperativ. (b) Infektion. (c) Freiliegende Platte nach Debridement. (d) Weichteildeckung durch M.gastrocnemius-Schwenklappenplastik. (e) Meshgraft-Deckung.

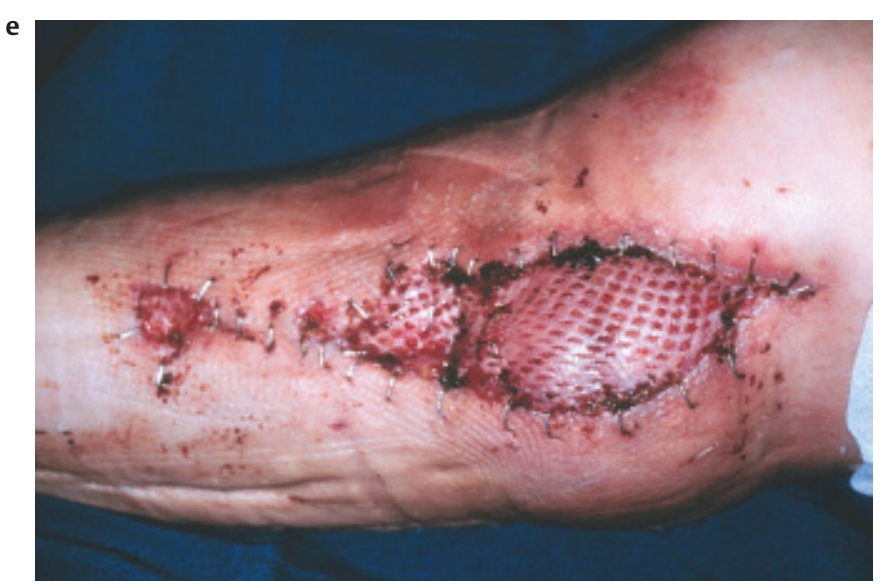



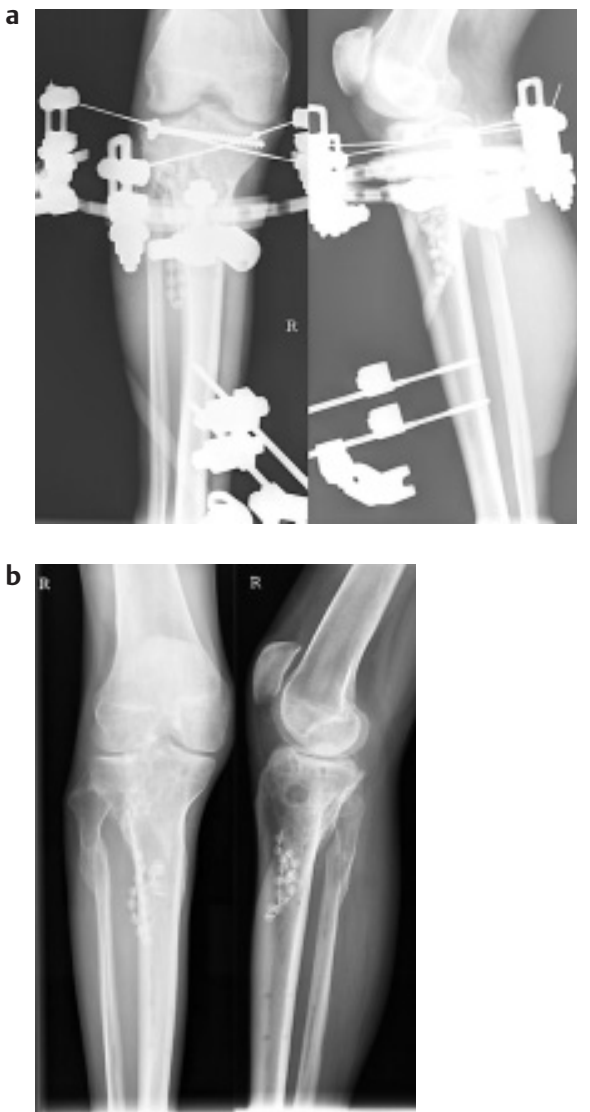

Abb. 2 Verfahrenswechsel nach infizierter Plattenosteosynthese bei prox. Tibiafraktur. (a) Hybridfixateur nach Plattenbettinfekt. (b) Ausheilung 2 Jahre nach Osteosynthese und Ausbehandlung im HybridFixateur externe.

gen, dass das primäre Ziel der Behandlung einer akuten postoperativen Implantatinfektion sein muss, die Infektsanierung zu erreichen, ohne damit eine chronische Osteitis zu verursachen. Hierbei hat sich das Operationskonzept bewährt, beim Entschluss zum Erhalt der Osteosynthese prinzipiell einen „second look“ nach 23 Tagen durchzuführen. Dabei gelten für das subtile Debridement, die Spülung und Abstrich sowie Gewebeproben dieselben Kriterien wie beim ersten Eingriff. Das Ziel, ein infektfreier Erhalt der Osteosynthese, wird dabei definitionsgemäß dann erreicht, wenn bei drei aufeinander folgenden operativen Revisionen Abstriche und Gewebeproben ohne Keimnachweis sind. Im Falle von weiter bestehender Kontamination nach 4 operativen Revisionen sollte das Osteosynthesematerial entfernt werden. Mit diesem Revisionskonzept konnten in einer prospektiven Studie mit Frühinfektion nach Osteosynthese $32 \%$ der Implantate erhalten werden [11].

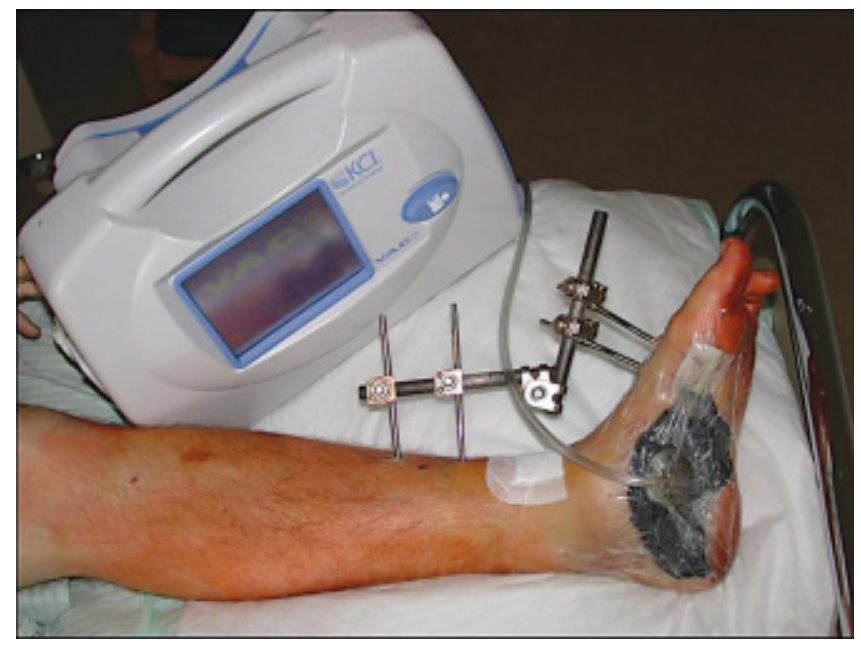

Abb. 3 Vakuumversiegelung.

Bei ausgedehnten Infekten mit schwerem Weichteilschaden und ungenügender Vaskularität im Fraktur- und Implantatbereich, aber auch bei ungenügender primärer Stabilität sind eine unmittelbare Materialentfernung und in der Regel externe Stabilisierung erforderlich (Abb. 2).

\section{Vitale Weichgewebedeckung}

Wie bereits mehrfach erwähnt, stellt die vitale Weichteildeckung eine absolute Grundvoraussetzung der erfolgreichen Infektionsbehandlung dar, welche lokal konditionierende Verfahren sowie die verschiedensten Möglichkeiten der chirurgisch-plastischen Rekonstruktion umfasst.

Bei ausgedehnterem Weichteilinfekt, fortgeschrittener Infektion und Implantatbelassung hat sich die Anlage einer Vakuumversiegelung [21] optimal bewährt.

Im eigenen Vorgehen werden dabei alle Bereiche der Wundhöhle mit den Schwämmen sorgfältig ausgelegt. Um eine Retraktion der Wundränder zu vermeiden und einen sicheren Unterdruck im Gewebe zu gewährleisten, wird die Haut über dem Schwamm vollständig verschlossen. Falls dies nicht möglich erscheint, wird der Schwamm zirkulär in den Hautdefekt eingenäht und luftdicht mit einer Obside-Folie versiegelt (Abb. 3).

Bei unzureichender Weichteildeckung nach Debridement stellt die frühe plastisch-chirurgische Rekonstruktion der Vaskularität einen weiteren entscheidenden Behandlungsschritt dar.

Dies kann in Abhängigkeit von der lokalen Infektionskontrolle und den struktu- rellen Gegebenheiten ein- oder zweizeitig (Überbrückung durch Vakuumversiegelung) erfolgen. Dabei kommen Jalousieplastiken und lokale Muskelschwenklappen sowie Fernlappen zur Anwendung, ggf. kombiniert mit primärer oder sekundärer Spalthaut (Abb. 2 und 4).

Eine alternative Möglichkeit stellt im Schaftbereich nach erforderlichem Defektdebridement die primäre Verkürzung der Extremität dar.

Dadurch können unter Umständen zum einen eine primär wesentlich höhere knöcherne Stabilität erreicht und ggf. aufwändigere plastisch-chirurgische Maßnahmen vermieden werden. Nach Fraktur- und Infektausheilung kann dann sekundär durch eine verlängernde Kallusdistraktion die ursprüngliche Situation wiederhergestellt werden.

\section{Adjuvante Antibiotikatherapie}

Eine „perioperative“ Therapie sollte im Gegensatz zu aseptischen Eingriffen erst intraoperativ nach Eröffnung der Wunde und Entnahme von Abstrichen und Gewebeentnahme durchgeführt werden.

Die antibiotische Therapie sollte systemisch prinzipiell keimgerecht nach Resistogramm erfolgen. Bei Nachweis von Staphylokokken als Erreger hat sich eine Kombination von Clindamycin und Rifampicin [8] oder Rifampicin und Oflaxacin [4] als effektiv erwiesen. Bis zum Erregernachweis werden in der Regel Cephalosporine der 2. oder 3. Generation, Breitspektrum Penicillin oder Clindamycin verwendet. 

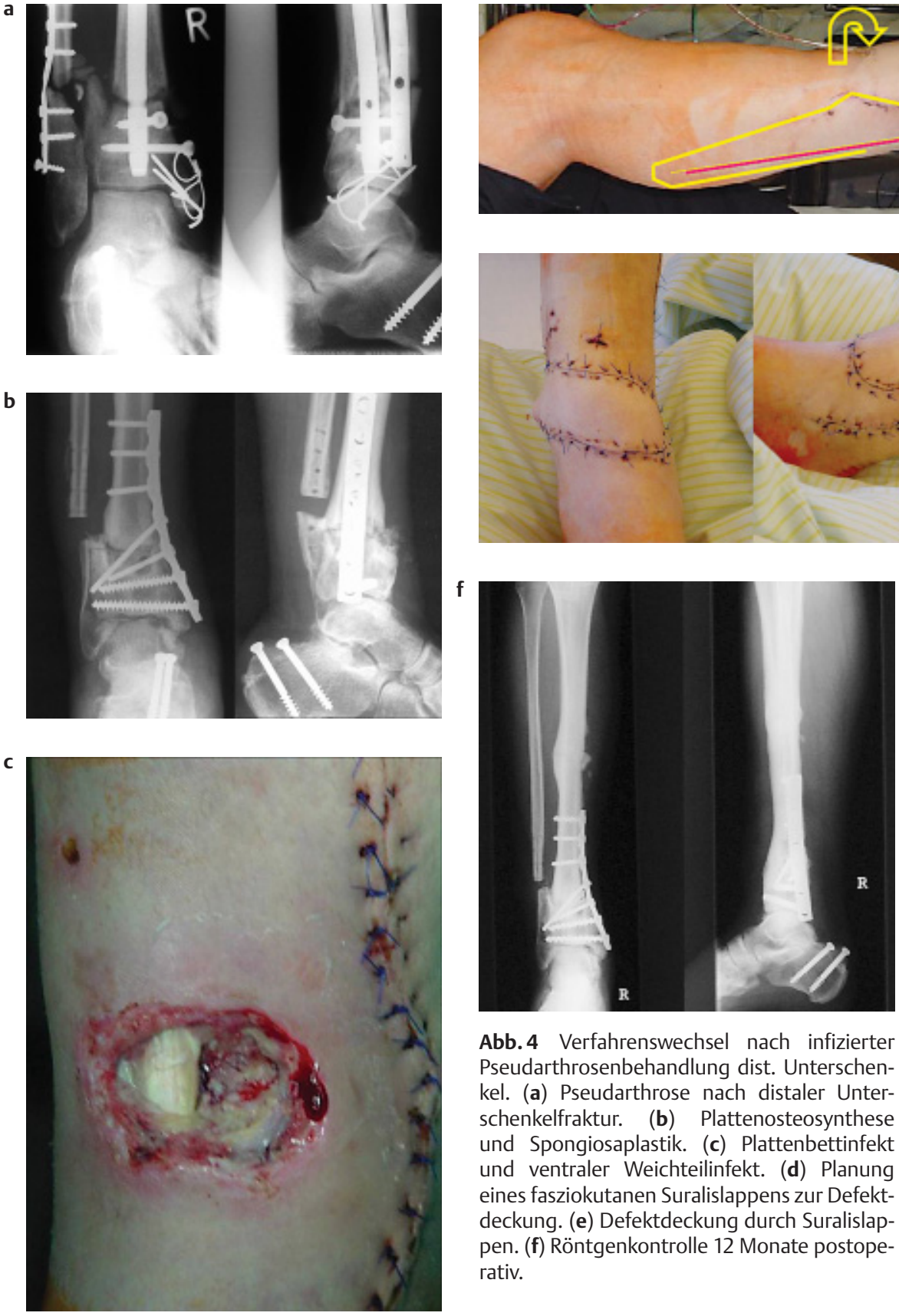

Abb. 4 Verfahrenswechsel nach infizierter Pseudarthrosenbehandlung dist. Unterschenkel. (a) Pseudarthrose nach distaler Unterschenkelfraktur. (b) Plattenosteosynthese und Spongiosaplastik. (c) Plattenbettinfekt und ventraler Weichteilinfekt. (d) Planung eines fasziokutanen Suralislappens zur Defektdeckung. (e) Defektdeckung durch Suralislappen. (f) Röntgenkontrolle 12 Monate postoperativ.

\section{Lokale Antibiotikaträger}

Es kann erwogen werden, Gentamicinketten oder resorbierbare gentamicinhaltige Kollagenschwämme lokal einzulegen [14].

Durch diese lokalen Antibiotikaträger werden lokale Wirkstoffkonzentrationen erreicht, die die Spiegel unter systemischer Therapie um das 200fache überschreiten. Dadurch können die Nebenwirkungen der systemischen Therapie vermieden und durch die extrem hohen Wirkstoffspiegel auch normalerweise resistente Keime noch erfolgreich elimi- niert werden [14]. Bei multiresistenten Keimen können auch mit Teicoplanin oder Vancomycin getränkte Kollagenschwämme oder PMMA-Ketten als lokale Antibiotikaträger eingesetzt werden [7]. Die Wirksamkeit konnte jedoch bisher in keiner einzigen kontrollierten Studie belegt werden [20].

In unserem Behandlungsvorgehen kommen diese Verfahren jedoch nur dann zur Anwendung, wenn Höhlen verbleiben und nicht durch lokale und freie Muskelschwenklappen mit vitalem Gewebe aufgefüllt werden können.

\section{Wundverschluss}

Redondrainagen sollten sowohl subfaszial um die Osteosynthese als auch in den subkutanen Bereich eingelegt werden. Kontinuierliche Saug-Spül-Drainagen werden nicht mehr empfohlen, da hierdurch eine Keimverschleppung in die Wunde stattfinden kann.

\section{Revisionseingriffe}

Wie bereits für „beim Implantat erhaltenden Vorgehen" dargestellt, kommt schon bei jedem Verdacht auf eine infizierte Osteosynthese der geplanten Revisionsoperation nach 2-3 Tagen eine entscheidende Bedeutung zu.

Ob weitere Folgeeingriffe stattfinden, hängt neben dem intraoperativen Befund im Wesentlichen vom neuerlichen Keimnachweis oder -ausschluss im Rahmen der Revisionsoperation ab.

Neben den bakteriologischen Befunden stellt auch der kontinuierliche Abfall der Infektparameter (insbesondere CRP) und deren Normalisierung ein weiteres wichtiges Kriterium zum Aussetzen weiterer geplanter Revisionseingriffe dar.

In Abhängigkeit von der primären Verletzung sowie dem Ausmaß und dem Verlauf der Infektion können neben den infektionsbedingten Revisionseingriffen weitere Verfahrenswechsel, auch unter Einbeziehung konservativer Stabilisierungsmethoden, zur Ausheilung der ursprünglichen Fraktur und der Wiederherstellung der Funktionalität erforderlich werden. Dies beinhaltet sowohl die Rekonstruktionen von Knochendefekten, 
sei es durch Spongiosaplastik, Kallusdistraktion oder Extremitätenverlängerung nach primärer Verkürzung bis hin zu rekonstruktiven Eingriffen an Muskeln und Sehnen bzw. plastisch-kosmetischen Maßnahmen.

\section{Schlussfolgerung}

Postoperative Infektionen lassen sich trotz absolut korrektem peri- und intraoperativen Vorgehen nicht vermeiden. Deshalb muss in Kenntnis dieses Risikos postoperativ eine lückenlose Überwachung erfolgen und dokumentiert werden. Ergeben sich dabei Hinweise auf einen Infekt, so gilt es diesen emotionslos schnellstmöglich auszuschließen oder zu bestätigen und unverzüglich adäquat $\mathrm{zu}$ therapieren. Dies bedeutet die einmalige oder meist wiederholte operative Revision mit sorgfältigem Debridement, ausgiebigem Spülen und die Gewährleistung der Stabilität der Osteosynthese, in Abhängigkeit vom Lokalbefund mit Implantatbelassen oder Verfahrenswechsel. Damit lassen sich viele Frühinfektionen direkt zur Ausheilung bringen, wohingegen bei ausgedehnten Infektionen zum Teil aufwendige ossäre und plastisch-chirurgische Verfahren zur Rekonstruktion erforderlich werden.

\section{Literatur}

${ }^{1}$ Atkins BL, Bowler IC. The diagnosis of large joint sepsis. J Hosp Infect 1998; 40: 263 -274

2 Bilat C, Leutenegger A, Ruedi T. Osteosynthesis of 245 tibial shaft fractures: early and late complications. Injury 1994; 25: 349-358

${ }^{3}$ Boriani S, Palmisani M, Donati U, Tella G, De Iure F, Bandiera S, Siccardi G. The treatment of thoracic and lumbar spine fractures: a study of 123 cases treated surgically in 101 patients. Chir Organi Mov 2000; 85: 137 149

${ }^{4}$ Drancourt M, Stein A, Argenson JN, Zannier A, Curvale G, Raoult D. Oral rifampin plus ofloxacin for treatment of Staphylococcus-infected orthopedic implants. Antimicrob Agents Chemother 1993; 37: 1214-1218

${ }^{5}$ Fitzgerald RH, Jr. Infected Total Hip Arthroplasty: Diagnosis and Treatment. J Am Acad Orthop Surg 1995; 3: 249-262

${ }^{6}$ Gerber A, Ganz R. Combined internal and external osteosynthesis a biological approach to the treatment of complex fractures of the proximal tibia. 1998; Injury 29 Suppl 3: $\mathrm{C} 22-28$

${ }^{7}$ Gerhart TN, Roux RD, Horowitz G, Miller RL, Hanff P, Hayes WC. Antibiotic release from an experimental biodegradable bone cement. J Orthop Res 1988; 6: 585-592

${ }^{8}$ Haas DW, McAndrew MP. Bacterial osteomyelitis in adults: evolving considerations in diagnosis and treatment. Am J Med 1996; 101: $550-561$

${ }^{9}$ Heinz T, Vecsei V. [Complications and errors in use of the gamma nail. Causes and prevention]. Chirurg 1994; 65: 943-952

${ }^{10}$ Hertel R, Pisan M, Lambert S, Ballmer FT. Plate osteosynthesis of diaphyseal fractures of the radius and ulna. 1996; Injury 27: $545-$ 548

${ }^{11}$ Hofmann GO, Bar T, Buhren V. (1997) [The osteosynthesis implant and early postoperative infection: healing with or without removal of the material?]. Chirurg 1996; 68: $1175-1180$

${ }^{12}$ Kampf G, Gastmeier P, Wischnewski N, Schlingmann J, Schumacher M, Daschner F, Ruden H. [Nosocomial infections in Germany-assessment and prevention. NIDEP Study, 1: On prevalence in surgery]. Chirurg 1996; 67: 637-642
${ }^{13}$ Kilian O, Bundner MS, Horas U, Heiss C, Schnettler R. [Long-term results in the surgical treatment of pilon tibial fractures. A retrospective study]. Chirurg 2002; 73: 65-72

${ }^{14} \mathrm{Klemm}$ K, Schnettler R. The use of gentamicin-PMMA chains in the treatment of infected tibial nonunion. Acta Orthop Belg 58 Suppl 1992; 1: $222-226$

${ }^{15}$ Neut D, van Horn JR, van Kooten TG, van der Mei HC, Busscher HJ. Detection of biomaterial-associated infections in orthopaedic joint implants. Clin Orthop 2003; 261-268

16 Pahud B, Vasey H. [Postoperative infection of fractures of the femoral diaphysis treated by osteosynthesis. Long-term outcome]. Rev Chir Orthop Reparatrice Appar Mot 1985; 71: $231-234$

${ }^{17}$ Rammelt S, Barthel S, Biewener A, Gavlik JM, Zwipp H. [Calcaneus fractures. Open reduction and internal fixation]. Zentralbl Chir 2003; 128: $517-528$

${ }^{18}$ Rüter A, Trentz O, Wagner M. Unfallchirurgie: Urban \& Fischer 2003: 251 -254

${ }^{19}$ Siebert CH, Heinz BC, Hofler HR, Hansis M. [Plate osteosynthesis management of humerus shaft fractures]. Unfallchirurg 1996; 99: $106-111$

${ }^{20}$ Stengel D, Bauwens K, Sehouli J, Ekkernkamp A, Porzsolt F. Systematic review and meta-analysis of antibiotic therapy for bone and joint infections. Lancet Infect Dis 2001; 1: 175-188

${ }^{21}$ Tautenhahn J, Burger T, Lippert H. [The present state of vacuum sealing]. Chirurg 2004 75: $492-497$

Prof. Dr. med. Dieter Nast-Kolb Ärztlicher Direktor Dr. med. Sascha Flohé Oberarzt Dr. med. Steffen Ruchholz Leitender Oberarzt

Klinik für Unfallchirurgie Universitätsklinikum Essen Hufelandstr. 55

D-45147 Essen 